\title{
GENERALIZED EVALUATION SUBGROUPS OF PRODUCT SPACES RELATIVE TO A FACTOR
}

\author{
KEE YOUNG LEE AND MOO HA WOO \\ (Communicated by Thomas Goodwillie)
}

\begin{abstract}
For any $C W$-complexes $X$ and $Y$, we show that $G_{n}(X \times Y, X)=$ $G_{n}(X) \oplus \pi_{n}(Y)$. We use this fact to compute generalized evaluation subgroups of generalized tori relative to a sphere.
\end{abstract}

\section{INTRODUCTION}

D.H.Gottlieb $[1,2]$ introduced the subgroups $G_{n}(X)$ of $\pi_{n}(X)$. These subgroups are the image of $\omega_{\sharp}: \pi_{n}\left(X^{X}, 1_{x}\right) \rightarrow \pi_{n}\left(X, x_{0}\right)$, where $X^{X}$ is the space of maps from $X$ to itself and $\omega: X^{X} \rightarrow X$ is the evaluation map. There is another equivalent definition of $G_{n}(X)$, i.e., $G_{n}(X)=\left\{[f] \in \pi_{n}(X) \mid \exists \operatorname{map} H: X \times I^{n} \longrightarrow X\right.$ such that $\left[\left.H\right|_{x_{0} \times I^{n}}\right]=[f]$ and $\left.H\right|_{X \times u}=1_{X}$ for $\left.u \in \partial I^{n}\right\}$. He used these groups to obtain some results about the identifications of topological spaces. Afterwards, many authors have studied and generalized $G_{n}(X)$, for instance, G.E.Lang, J.Siegel, K.L.Lim, M.H.Woo and J.Kim, Varadarajian and N.Oda in $[5,7,8,9,12]$, etc. In [12], the second author and J. Kim have generalized $G_{n}(X)$ to $G_{n}(X, A)$. These subgroups are defined by the image $\omega_{\sharp}: \pi_{n}\left(X^{A}, i\right) \rightarrow \pi_{n}\left(X, x_{0}\right)$, where $X^{A}$ is the space of maps from $A$ to $X, i: A \rightarrow X$ is the inclusion and $\omega: X^{A} \rightarrow X$ is the evaluation map. Equivalently, $G_{n}(X, A)=\left\{[f] \in \pi_{n}(X) \mid \exists \operatorname{map} H: A \times I^{n} \longrightarrow X\right.$ such that $\left[\left.H\right|_{x_{0} \times I^{n}}\right]=[f]$ and $\left.H\right|_{A \times u}=i$ for $\left.u \in \partial I^{n}\right\} . G_{n}(X, A)$ always contains $G_{n}(X)$ and $G_{n}(X, A)=G_{n}(X)$ if $A=X, G_{n}(X, A)=\pi_{n}(X)$ if $A=x_{0}$. In [6, 13], the authors introduced the subgroups $G_{n}^{R e l}(X, A)$ of the relative homotopy groups $\pi_{n}(X, A)$ which are defined by the image $\omega_{\sharp}: \pi_{n}\left(X^{A}, A^{A}, i\right) \rightarrow \pi_{n}\left(X, A, x_{0}\right)$, where $A^{A}$ is the subspace of $X^{A}$ which consists of maps from $A$ into itself. Equivalently, $G_{n}^{\text {Rel }}(X, A)=\left\{[f] \in \pi_{n}(X, A) \mid \exists \operatorname{map} H:\left(X \times I^{n}, A \times \partial I^{n}\right) \longrightarrow(X, A)\right.$ such that $\left[\left.H\right|_{x_{0} \times I^{n}}\right]=[f]$ and $\left.H\right|_{X \times u}=1_{X}$ for $\left.u \in J^{n-1}\right\}$. In [6,13], we showed that for a CW-pair $(X, A), G_{n}(A), G_{n}(X, A)$, and $G_{n}^{R e l}(X, A)$ fit together into a sequence

$$
\begin{aligned}
\cdots \rightarrow G_{n}(A) \stackrel{i_{\sharp}}{\longrightarrow} G_{n}(X, A) \stackrel{j_{\sharp}}{\longrightarrow} G_{n}^{R e l}(X, A) \\
\stackrel{\partial}{\rightarrow} \cdots \rightarrow G_{1}^{R e l}(X, A) \rightarrow G_{0}(A) \rightarrow G_{0}(X, A),
\end{aligned}
$$

Received by the editors January 25, 1995.

1991 Mathematics Subject Classification. Primary 55P45.

Key words and phrases. Generalized evaluation subgroup, $G$-sequence of the trivial fibration, trivial fibration.

Partially supported by TGRC-KOSEF and BSRI 94-1409. 
where $i_{\sharp}, j_{\sharp}$ and $\partial$ are restrictions of the usual homomorphisms of the homotopy sequence

$$
\cdots \stackrel{\partial}{\rightarrow} \pi_{n}(A) \stackrel{i_{\sharp}}{\longrightarrow} \pi_{n}(X) \stackrel{j_{\sharp}}{\longrightarrow} \pi_{n}(X, A) \rightarrow \cdots \rightarrow \pi_{0}(A) \longrightarrow \pi_{0}(X) .
$$

This sequence is called the $G$-sequence of a $\mathrm{CW}$-pair $(X, A)$. In $[6,13]$, we showed that in general, it is not exact; but it is exact when the inclusion $i: A \rightarrow X$ has a left homotopy inverse or is homotopic to a constant map.

The subgroups $G_{n}(X), G_{n}(X, A)$ and $G_{n}^{R e l}(X, A)$ mentioned above are called evaluation subgroups, generalized evaluation subgroups and relative evaluation subgroups respectively. Only a few computations of evaluation subgroups are known despite the fact that several authors have studied them. In order to find nice applications to identifications of spaces or to the fixed point theory, we need to compute many evaluation subgroups. When the second author used $G_{n}(X, A)$ to obtain some results of the Nielsen fixed point theory, we needed the computations of $G_{n}(X, A)$ which are proper subgroups of $\pi_{n}(X)$ and contain $G_{n}(X)$ properly, i.e., $G_{n}(X) \varsubsetneqq G_{n}(X, A) \varsubsetneqq \pi_{n}(X)$. In this paper, we prove the following theorem which gives us many examples such that $G_{n}(X) \varsubsetneqq G_{n}(X, A) \varsubsetneqq \pi_{n}(X)$. We shall call such a subgroup $G_{n}(X, A)$ a proper generalized evaluation subgroup.

Theorem. Let $F \stackrel{i}{\rightarrow} F \times B \stackrel{p}{\rightarrow} B$ be a trivial fibration. Then we have

$$
G_{n}(F \times B, F) \cong \pi_{n}(B) \oplus G_{n}(F) .
$$

As corollaries, we look for the necessary and sufficient condition for the product space $X \times Y$ to have proper generalized evaluation subgroups $G_{n}(X \times Y, X)$ relative to $X$ and we compute several proper generalized evaluation subgroups.

Throughout this paper, all spaces are $C W$-complexes and all topological pairs are $C W$-pairs.

\section{Proof of the theorem}

Let $p: F \times B \rightarrow B$ be a trivial fibration. $p$ induces a homomorphism $p_{\sharp}$ : $\pi_{n}(F \times B, F) \rightarrow \pi_{n}(B)$ which is one to one and onto. In order to prove the theorem, we first introduce an exact sequence similar to homotopy sequence of a fibration.

Lemma. The restriction $\left.p_{\sharp}\right|_{G_{n}^{R e l}(F \times B, F)}: G_{n}^{R e l}(F \times B, F) \rightarrow \pi_{n}(B)$ is one to one and onto.

Proof. Since the map $p$ induces the bijective homomorphism $p_{\sharp}: \pi_{n}(F \times B, F) \rightarrow$ $\pi_{n}(B)$, it is sufficient to show that $\left.p_{\sharp}\right|_{G_{n}^{R e l}(F \times B, F)}$ is onto. Let $[f]$ be any element of $\pi_{n}(B)$. Then $f:\left(I^{n}, \partial I^{n}\right) \rightarrow\left(B, b_{0}\right)$ is a continuous map of pairs. Define

$$
H: F \times B \times I^{n-1} \times 0 \sqcup F \times b_{0} \times I^{n-1} \times I \rightarrow B
$$

by

$$
H(x, b, u, 0)=b \text { and } H\left(x, b_{0}, u, t\right)=f h(u, t)
$$


where $(x, b, u, 0) \in F \times B \times I^{n-1} \times 0,\left(x, b_{0}, u, t\right) \in F \times b_{0} \times I^{n-1} \times I$ and $h:$ $I^{n} \rightarrow I^{n}$ is a homeomorphism such that $h\left(J^{n-1}\right)=I^{n-1} \times 0$ and $h h=1$. Since $\left(F \times B \times I^{n-1}, F \times b_{0} \times I^{n-1}\right)$ is a $C W$-pair, there is an extension $\bar{H}: F \times B \times$ $I^{n-1} \times I \rightarrow B$ by the absolute homotopy extension property. Define

$$
\theta: F \times B \times I^{n-1} \times I \rightarrow F \times B
$$

by

$$
\theta(x, b, u, t)=(x, \bar{H}(x, b, h(u, t))) .
$$

Then $\theta$ is well-defined and continuous. Moreover, $p \theta\left(x_{0}, b_{0}, u, t\right)=f(u, t)$, where $x_{0}$ is a base point of $F, \theta(x, b, u, s)=(x, b)$ for $(x, b, u, s) \in F \times B \times J^{n-1}$ and $\theta\left(x, b_{0}, u, t\right)=\left(x, b_{0}\right) \subset F \times b_{0}$ for $\left(x, b_{0}, u, t\right) \in F \times b_{0} \times \partial I^{n}$. Let

$$
\alpha=\left.\theta\right|_{\left(x_{0}, b_{0}\right) \times I^{n}}:\left(I^{n}, \partial I^{n}, J^{n-1}\right) \rightarrow\left(F \times B, F \times b_{0},\left(x_{0}, b_{0}\right)\right) .
$$

Then

$$
[\alpha]=\left[\left.\theta\right|_{\left(x_{0}, b_{0}\right) \times I^{n}}\right] \in G_{n}^{R e l}(F \times B, F)
$$

because $\theta$ is an associated map of $\alpha$. Since $p \theta\left(x_{0}, b_{0}, u, t\right)=f(u, t)$,

$$
p_{\sharp}[\alpha]=[p \alpha]=\left[\left.p \theta\right|_{\left(x_{0}, b_{0}\right) \times I^{n}}\right]=[f] .
$$

So we have $[f] \in p_{\sharp}\left(G_{n}^{R e l}(F \times B, F)\right)$.

Consider the following commutative diagram:

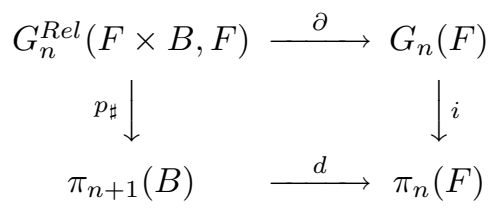

where $\partial$ is the boundary homomorphism in the homotopy sequence of the pair $(E \times F, F)$ and $d$ is the connecting homomorphism in the homotopy sequence of the fibration $F \stackrel{i}{\rightarrow} F \times B \stackrel{p}{\rightarrow} B$. Since $p_{\sharp}$ is a bijective map, we have

$$
d\left(\pi_{n+1}(B)\right)=i \partial p_{\sharp}^{-1}\left(\pi_{n+1}(B)\right)=i \partial\left(G_{n+1}^{R e l}(F \times B, F)\right) \subseteq i\left(G_{n}(F)\right)=G_{n}(F) .
$$

Thus we have the following sequence which is a chain complex:

$$
\begin{aligned}
\cdots \rightarrow G_{n}(F) & \stackrel{i_{\sharp}}{\longrightarrow} G_{n}(F \times B, F) \stackrel{p_{\sharp}}{\longrightarrow} \pi_{n}(B) \\
& \stackrel{d}{\longrightarrow} \cdots \rightarrow G_{0}(F) \rightarrow G_{0}(F \times B, F) \rightarrow \pi_{0}(B) .
\end{aligned}
$$

This sequence is called the $G$-sequence of the trivial fibration.

Proof of the theorem. It is shown in [13, Theorem 11] that if the inclusion $i: A \rightarrow X$ has a left homotopy inverse, then the $G$-sequence of the pair $(X, A)$ is exact. Let $\pi_{1}: F \times B \rightarrow F$ be the projection to first coordinate. Then $\pi_{1}$ is a left homotopy 
inverse of $i: F \rightarrow F \times B$. So the $G$-sequence of the pair $(F \times B, F)$ is exact. Consider the following commutative diagram:

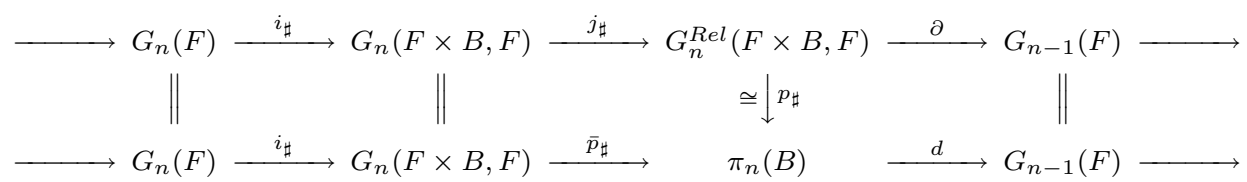

where $\bar{p}_{\sharp}=p_{\sharp} \circ j_{\sharp}$ and $d=\partial \circ p_{\sharp}^{-1}$. Since the $G$-sequence of the pair $(F \times B, F)$ is exact and $p_{\sharp}$ is an isomorphism, the $G$-sequence of the trivial bundle is also exact. Since $i_{\sharp}$ is a monomorphism, we have $d\left(\pi_{n}(B)\right)=0$ and $p_{\sharp}$ is an epimorphism. Now we will show the homomorphism $p_{\sharp}: \pi_{n}(F \times B) \rightarrow \pi_{n}(F)$ induces a homomorphism $p_{\sharp}: G_{n}(F \times B, F) \rightarrow G_{n}(F)$. Let $[f]$ be an element of $G_{n}(F \times B, F)$. Then there exists a homotopy $H: F \times I^{n} \rightarrow F \times B$ such that

$$
\left[\left.H\right|_{x_{0} \times I^{n}}\right]=[f] \text { and }\left.H\right|_{F \times u}=i \text { for } u \in \partial I^{n} .
$$

If we define $\bar{H}=p H: F \times I^{n} \rightarrow F$, then we have

$$
\left[\left.\bar{H}\right|_{x_{0} \times I^{n}}\right]=[p f] \text { and }\left.\bar{H}\right|_{F \times u}=i d \text { for } u \in \partial I^{n} .
$$

Therefore $p_{\sharp}([f])$ belongs to $G_{n}(F)$ and hence $p_{\sharp}$ induces a homomorphism $p_{\sharp}$ : $G_{n}(F \times B, F) \rightarrow G_{n}(F)$. If we define a homomorphism $\phi: G_{n}(F \times B, F) \rightarrow \pi_{n}(B) \oplus$ $G_{n}(F)$ by $\phi(\alpha)=\left(p_{\sharp}(\alpha), \pi_{1 \sharp}(\alpha)\right)$, it is easy to show that $\phi$ is an isomorphism. Thus the theorem is proved.

\section{Computations of Generalized EVAluation SubGroups}

Corollary 1. For any spaces $X$ and $Y, G_{n}(X \times Y, Y) \cong \pi_{n}(X) \oplus G_{n}(Y)$ for all $n$.

Proof. Since $X \stackrel{i}{\rightarrow} X \times Y \stackrel{\pi_{2}}{\longrightarrow} Y$ is a trivial fibration, it follows from the theorem .

Corollary 1 enables us to find many $C W$-pairs $(X, A)$ such that $G_{n}(X) \varsubsetneqq$ $G_{n}(X, A) \varsubsetneqq \pi_{n}(X)$. Such a pair $(X, A)$ is said to have the proper $n$-th generalized evaluation subgroup.

Corollary 2. The pair $(X \times Y, Y)$ has the proper $n$-th generalized evaluation subgroup if and only if $G_{n}(X)$ is a proper subgroup of $\pi_{n}(X)$ and $G_{n}(Y)$ is a proper subgroup of $\pi_{n}(Y)$.

Proof. It follows from the following relations

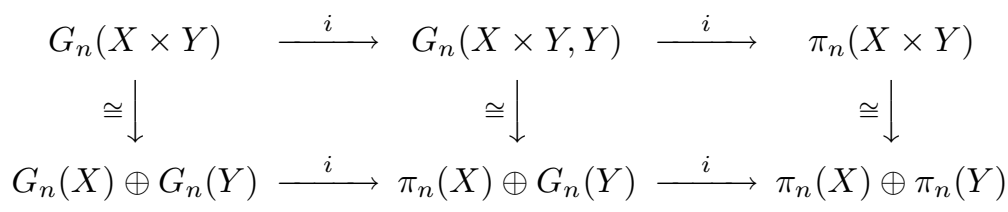

where the first and the second isomorphisms are restrictions of the third one and $i$ 's are inclusions. 
Corollary 3. The pair $(X \times X, X)$ has the proper $n$-th generalized evaluation subgroup if and only if $G_{n}(X)$ is a proper subgroup of $\pi_{n}(X)$.

Example 1. If $n$ is even, then $0=G_{n}\left(S^{n}\right) \varsubsetneqq \pi_{n}\left(S^{n}\right)$. So $\left(S^{n} \times S^{n}, S^{n}\right)$ has the proper $n$-th generalized evaluation subgroup. More precisely,

$$
G_{n}\left(S^{n} \times S^{n}\right)=G_{n}\left(S^{n}\right) \oplus G_{n}\left(S^{n}\right)=0 \varsubsetneqq G_{n}\left(S^{n} \times S^{n}, S^{n}\right)=\mathbf{Z} \varsubsetneqq \pi_{n}\left(S^{n} \times S^{n}\right)=\mathbf{Z} \oplus \mathbf{Z} .
$$

If $n$ is odd and $n \neq 1,3,7$, then $G_{n}\left(S^{n}\right)=2 \mathbf{Z} \varsubsetneqq \pi_{n}\left(S^{n}\right)$. So $\left(S^{n} \times S^{n}, S^{n}\right)$ has the proper $n$-th generalized evaluation subgroup. More precisely,

$$
G_{n}\left(S^{n} \times S^{n}\right)=2 \mathbf{Z} \oplus 2 \mathbf{Z} \varsubsetneqq G_{n}\left(S^{n} \times S^{n}, S^{n}\right)=\mathbf{Z} \oplus 2 \mathbf{Z} \varsubsetneqq \pi_{n}\left(S^{n} \times S^{n}\right)=\mathbf{Z} \oplus \mathbf{Z} .
$$

If we use Corollary 3 and the computations of $\pi_{m}\left(S^{n}\right)$ and $G_{m}\left(S^{m}\right)$, then we can compute generalized evaluation subgroups of the pairs $\left(S^{n} \times S^{m}, S^{m}\right)$.

By the fact that $\pi_{n+1}\left(S^{n}\right)$ is cyclic of order 2 for every $n \geqq 3$ and computations of $G_{n}\left(S^{n}\right)$, we have the following.

\section{Example 2.}

$$
G_{n+1}\left(S^{n} \times S^{n+1}, S^{n+1}\right) \cong \begin{cases}0, & n=1 \\ \mathbf{Z} \oplus \mathbf{Z}, & n=2, \\ \mathbf{Z}, & n=3, \\ \mathbf{Z}_{2}, & n>3 \text { and } n \text { is odd } \\ \mathbf{Z}_{2} \oplus \mathbf{Z}, & n=6, \\ \mathbf{Z}_{2} \oplus 2 \mathbf{Z}, & n>3 \text { and } n \text { is even }\end{cases}
$$

By the fact that $\pi_{n+2}\left(S^{n}\right)$ is cyclic of order 2 for every $n \geqq 3$ and computations of $G_{n+2}\left(S^{n+2}\right)$, we have the following.

\section{Example 3.}

$$
G_{n+2}\left(S^{n} \times S^{n+2}, S^{n+2}\right) \cong \begin{cases}\mathbf{Z}, & n=1 \\ \mathbf{Z}, & n=2, \\ \mathbf{Z}_{2} \oplus 2 \mathbf{Z}, & n=3, \\ \mathbf{Z}_{2}, & n>3 \text { and } n \text { is even } \\ \mathbf{Z}_{2} \oplus \mathbf{Z}, & n=5, \\ \mathbf{Z}_{2} \oplus 2 \mathbf{Z}, & n>3 \text { and } n \text { is odd. }\end{cases}
$$

By the computations of $\pi_{n+3}\left(S^{n}\right)$ and $G_{n+3}\left(S^{n+3}\right)$, we have the following.

\section{Example 4.}

$$
\begin{aligned}
& G_{6}\left(S^{3} \times S^{6}, S^{6}\right) \cong \mathbf{Z}_{12}, \\
& G_{7}\left(S^{3} \times S^{7}, S^{7}\right) \cong \mathbf{Z} \oplus \mathbf{Z}_{12} \oplus \mathbf{Z}, \\
& G_{n+3}\left(S^{n} \times S^{n+3}, S^{n+3}\right) \cong \begin{cases}\mathbf{Z}_{24} \oplus 2 \mathbf{Z}, & \text { if } n \geqq 5 \text { and } n \text { is even, } \\
\mathbf{Z}_{24}, & \text { if } n \geqq 5 \text { and } n \text { is even. }\end{cases}
\end{aligned}
$$

By the computations of $\pi_{n+4}\left(S^{n}\right)$ and $G_{n+4}\left(S^{n+4}\right)$, we have the following. 
Example 5.

$$
\begin{aligned}
& G_{7}\left(S^{3} \times S^{7}, S^{7}\right) \cong \mathbf{Z}_{2} \oplus \mathbf{Z}, \\
& G_{8}\left(S^{4} \times S^{8}, S^{8}\right) \cong \mathbf{Z}_{2}, \\
& G_{9}\left(S^{5} \times S^{9}, S^{9}\right) \cong \mathbf{Z}_{2} \oplus 2 \mathbf{Z}, \\
& G_{n+4}\left(S^{n} \times S^{n+4}, S^{n+4}\right) \cong \begin{cases}0, & \text { if } n \text { is even }, \\
2 \mathbf{Z}, & \text { if } n \text { is odd } .\end{cases}
\end{aligned}
$$

\section{REFERENCES}

1. D. H. Gottlieb, A certain subgroup of the fundamental group, Amer. J. Math. 87 (1965), 840-856. MR 32:6454

2. $ـ$ Evaluation subgroups of homotopy groups, Amer. J. Math. 91 (1969), 729-756. MR 43:1181

3. Covering transformations and universal fibrations, Illinois J. Math.13(1969),432-437. MR 39:952

4. B. Gray, Homotopy Theory, Academic press, New York, 1975. MR 53:6528

5. G. E. Lang, Jr., Evaluation subgroups of factor spaces, Pacific J. Math. 42 (1972), 701-709. MR 47:2595

6. K.Y. Lee and M.H. Woo, G-sequences and $\omega$-homology of a $C W$-pair, Topology and its application 52 (1993), 221-236. MR 94i:55016

7. K.L. Lim, On cyclic maps, J. Austral. Math. Soc. Ser. A, 32 (1982), 349-357. MR 83e:55003

8. N. Oda, The homotopy set of the axes of pairings, Canad. J. Math. 42 (1990), 856-868.

9. J. Siegel, G-spaces, W-spaces and H-spaces, Pacific J. Math. 31 (1969), 209-214. MR 48:4950

10. K. Varadarajian, Generalized Gottlieb Groups, J. Indian Math. Soc. 33 (1969), 141-164. MR 43:6926

11. G. W. Whitehead, Elements of homotopy theory, Springer-Verlag, New York, 1978. MR 80b:55001

12. M. H. Woo and J. R. Kim, Certain subgroups of homotopy groups, J. of Korean Math. Soc. 21 (1984), 109-120. MR 86c:55014

13. M. H. Woo and K. Y. Lee, The relative evaluation subgroups of a $C W$-pair, J. of Korean Math. Soc. 25 (1988), 149-160. MR 89f:55011

14. - Homology and generalized evaluation subgroups of homotopy groups, J. of Korean Math. Soc. 25 (1988), 333-342. MR 89k:55026

Department of Mathematics, Taejon National University of Technology, Taejon 300, KoreA

Department of Mathematics Education, Korea University, Seoul 136, Korea 